\title{
Final Technical Report \\ Grain Boundary Complexions and Transitions in Doped Silicon
}

\section{Award \#: DE-FG02-08ER46511}

\author{
Submitted by \\ Jian Luo \\ Professor \\ Department of Materials Science and Engineering \\ Clemson University \\ Olin Hall 206, Clemson, SC 29634 \\ Phone: (864) 656-5961 \\ Fax: (864) 656-1453 \\ E-mail: jianluo@clemson.edu
}

Date: October 15, 2012

Submitted to

Dr. Jane G. Zhu

Program Manager

Electron and Scanning Probe Microscopies

Division of Materials Sciences and Engineering

Office of Basic Energy Sciences

U.S. Department of Energy

SC-22.2/Germantown Building

1000 Independence Avenue, SW

Washington, DC 20585-1290

Phone: (301) 903-3811

Fax: (301) 903-9513

E-mail: Jane.Zhu@science.doe.gov

Cheryl L. Howard, SC-22.2

Program Assistant

Office of Basic Energy Science

U.S. Department of Energy

Germantown Building

1000 Independence Avenue, SW

Washington, DC 20585-1290

Phone: (301) 903-3428

Fax: (301) 903-9515

E-mail: Cheryl.Howard@ science.doe.gov 


\section{DOE award \# and name of the recipient}

- DOE award \#: DE-FG02-08ER46511

- Recipient: Clemson University

\section{Project title and name of the PI}

- Title: Grain Boundary Complexions and Transitions in Doped Silicon

- PI: Jian Luo

\section{Date of the report and period covered by the report}

- Date of the Report: October 15, 2012

- Period Covered: July 15, 2008 to July 14, 2012

\section{Abstract}

This four-year research project has advanced the fundamental knowledge of grain boundary (GB) complexions (i.e., "two-dimensional interfacial phases") and associated GB "phase" transitions in several grounds. First, a bilayer interfacial phase, which had been directly observed by microscopy only in complex ceramic systems in prior studies, has been identified in simpler systems such as Au-doped $\mathrm{Si}$ and Bi-doped Ni in this study, where the interpretations of the their formation mechanisms and microscopic images are less equivocal. Second, convincing evidence for the existence of a first-order GB transition from a nominally "clean" GB to a bilayer adsorption interfacial phase has been revealed for Au-doped Si; the confirmation of the firstorder nature of interfacial transitions at GBs, which was rare in prior studies, is scientifically significant and technologically important. Third, the bilayer interfacial phase discovered in Bidoped $\mathrm{Ni}$ has been found to be the cause of the mysterious liquid metal embrittlement phenomenon in this system; the exact atomic level mechanism of this phenomenon has puzzled the materials and physics communities for over a century. Finally, significant advancements have been made to establish phenomenological thermodynamic models for GB complexions and transitions. Since GB complexions can control the transport, mechanical and physical properties of a broad range of metallic and ceramic materials, the fundamental knowledge generated by this project can have broad impacts on materials design in general. In this regard, understanding and controlling GB phase behaviors (complexions and transitions) can be an important component for the "Materials Genome" project. 


\section{Description of accomplishment}

\section{(A) Background and Introduction}

The concept that interfaces such as grain boundaries (GBs) can exhibit "phase" behaviors is not new. In 1968 and 1972, Hart already proposed that GBs can be considered as "two-dimensional interfacial phases" that may undergo "phase" transformations $[1,2]$. Subsequent thermodynamic models developed by Cahn [3-6], Clarke [7], Tang, Carter and Cannon [8, 9], Wynblatt and Chatain [10, 11], and Luo [A6] further elaborated this concept. Tang, Carter and Cannon [8, 9] first introduced the terminology "complexion"; they argued that an interfacial complexion is not a "phase" according to Gibbs' rigorous definition because it has no identifiable volume and it cannot exist alone without abutting bulk phase(s).

One particular "liquid-like" GB complexion is represented by impurity-based equilibrium-thickness intergranular films (IGFs), which were widely observed in structural and functional ceramics [7, 12, 13]. Interfacial films of similar character have been found at metallic GBs [14-16], as well as at metal-oxide interfaces [17-19] and on free surfaces [20-22]. These nanoscale IGFs often control microstructural development and physical properties [12]. In 2007, an important advancement was reported by Dillon $e t$ al. [23-26], who discovered a series of distinct GB complexions in alumina: an intrinsic-like "clean" GB, a monolayer, a bilayer, a trilayer, a nanoscale IGF, and a wetting film (Fig. 1).
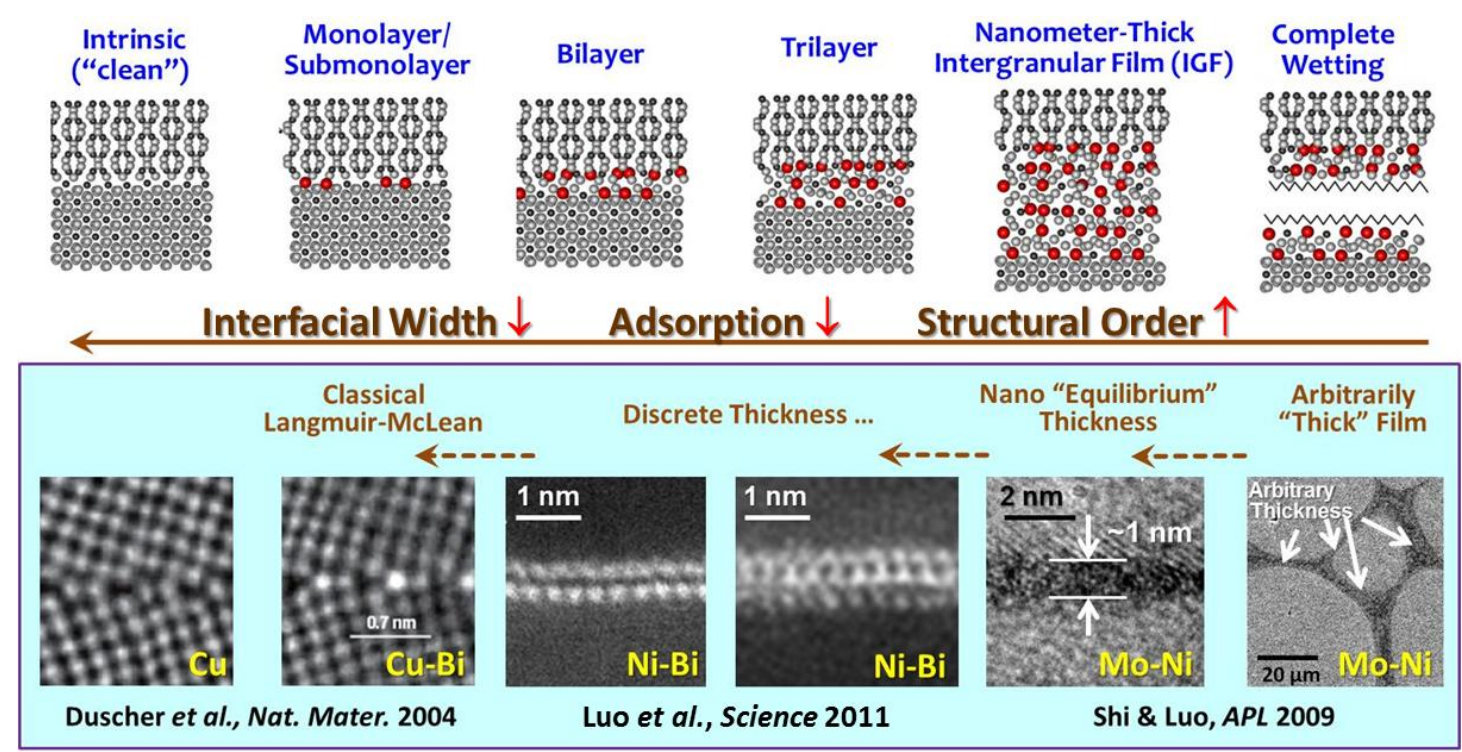

Figure 1: Schematic illustration of a series of complexions that were initially observed in doped $\mathrm{Al}_{2} \mathrm{O}_{3}$ by Dillon and Harmer [23, 24]. Similar complexions were also observed in various metallic systems [A4]. Recent observations of the most controversial bilayer complexion and an associated first-order GB transition in $\underline{\mathrm{Si}}-\mathrm{Au}$ [A2], as well as a series of three GB complexion transitions in $\underline{\mathrm{TiO}}_{2}-\mathrm{CuO}-\mathrm{SiO}_{2}$ [A1], further demonstrated the generality and diversity of GB complexion formations and transitions.

In collaboration with a Lehigh team led by Prof. Harmer, in this DOE-BES project we have made a series of important experimental discoveries of complexion formations and transitions in $\underline{\mathrm{Si}}-\mathrm{Au}$ (i.e., $\mathrm{Au}-$ doped $\mathrm{Si}$ ) [A2] and Ni-Bi [A3, A4] (which were the proposed primary and secondary model systems, respectively, in our original proposal). Furthermore, we have made advancements in establishing general interfacial thermodynamic models for GB complexion formation and transition [A5-A9] and helped the Lehigh team to interpret their observation of complexions and transitions in $\mathrm{TiO}_{2}-\mathrm{CuO}-\mathrm{SiO}_{2}[\mathrm{~A} 1]$. Key achievements are described subsequently. 


\section{(B) Development of Thermodynamic Models}

In the theoretical and modeling thrust, we have developed a thermodynamic model for coupled adsorption and disordering transitions at GBs by combining diffuse-interface and lattice-gas models [A6, A7]. This interfacial thermodynamic model considers 1) the through-thickness gradients, 2) a simplified atomic size effect (assuming that atoms are represented by structure-less hard spheres via adopting a "solvation" interaction), and 3) the effects of additional interfacial interactions (such as van der Waals London dispersion and electrostatic interactions). Fig. 2(a) schematically illustrates the key steps in constructing this model; further details and formulation can be found in our DOE supported research publication in Appl. Phys. Lett. [A6] and a subsequent invited overview article in JOM [A7].
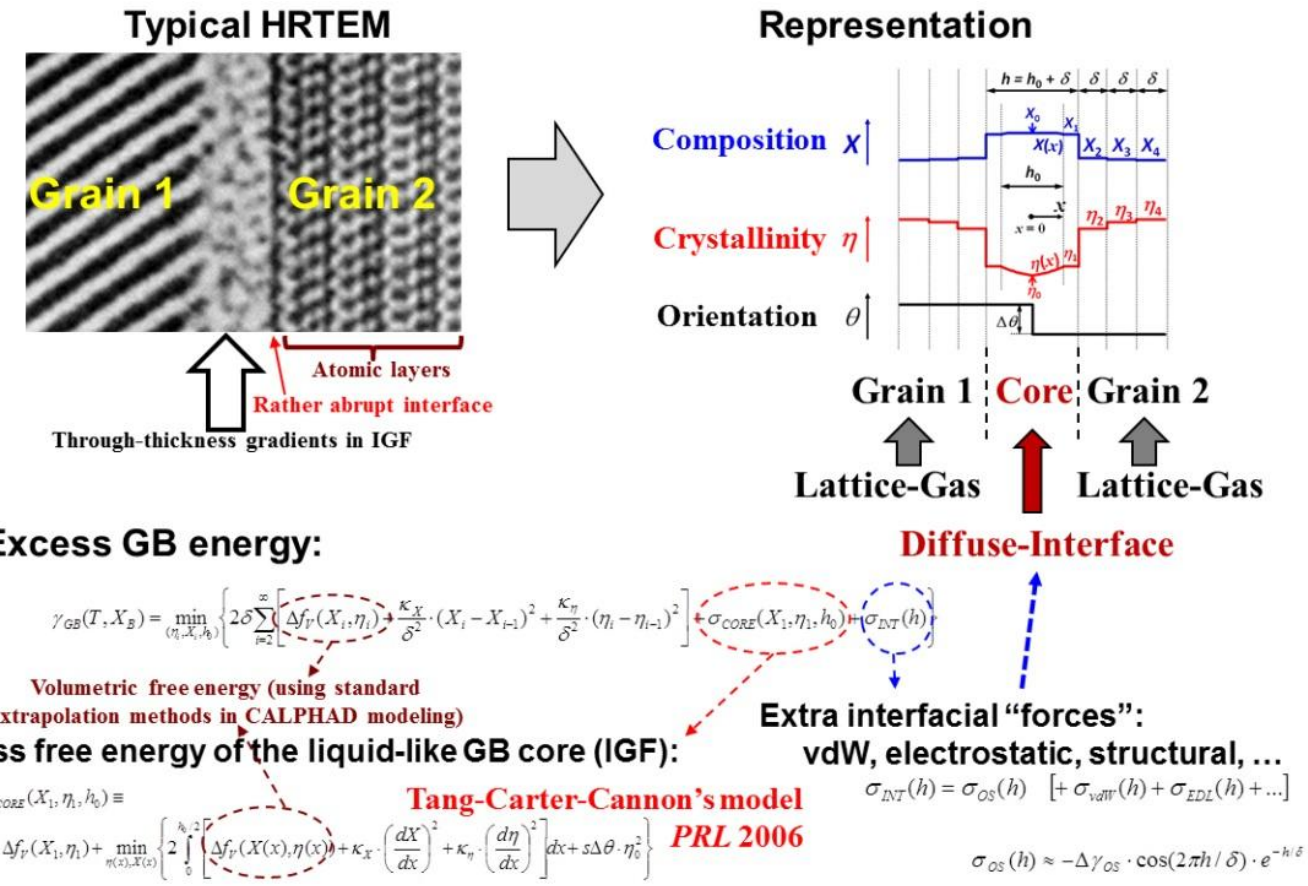

(a)

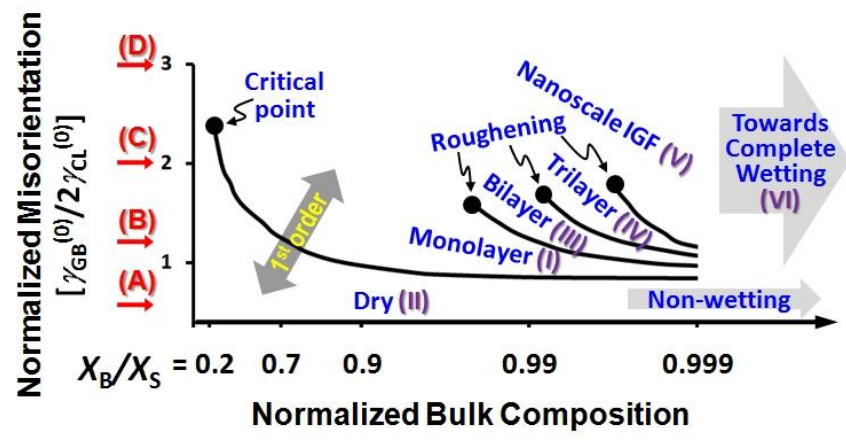

(b)

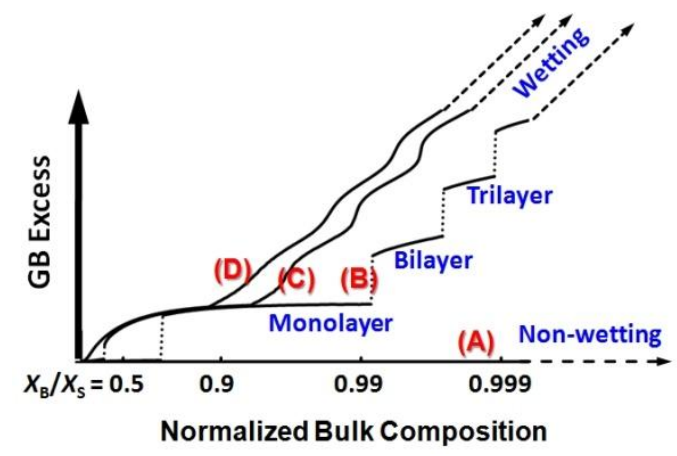

(c)

Figure 2: (a) Schematic illustration of an interfacial thermodynamic model developed in this BES project by combining a diffuse-interface model with a lattice-gas model and incorporating extra colloidal-type interfacial interactions. (b) A representative GB complexion (phase) diagram, in which first-order transition lines and critical points are plotted in the field of normalized bulk composition and normalized GB misorientation. (c) Computed GB excess vs. normalized bulk composition for four selected GBs that are indicated by the arrows in (b). (Figure adapted from Ref. [A6]) 
This thermodynamic model produces a systematical spectrum of interfacial phenomena for GBs, including first-order and continuous coupled prewetting and premelting transitions, critical points, multilayer adsorption, layering and roughening, and complete wetting and drying; it produces a series of generic GB complexions with character similar to those observed by Dillon and Harmer [Fig. 2 (a) and (b)]. We should emphasize that such GB phase behaviors are analogous to a case of multilayer gas adsorption on an attractive inert substrate, for which similar surface phase diagrams had been constructed by physicists in 1980s [27]; this analogue helps us to understand the physical origin of this series of GB complexions. We also demonstrated that the presence of London dispersion and electrostatic forces in ceramic materials could appreciably change the GB phase behaviors [A6].

Via the support of our BES grant, we also collaborated with Lehigh researchers to explain their observations in $\mathrm{a} \mathrm{TiO}_{2}$ bicrystal. The Lehigh team made a very interesting observation of the formation of a series of GB complexions in the $\mathrm{TiO}_{2}-\mathrm{CuO}-\mathrm{SiO}_{2}$ system, including a monolayer, a bilayer, a trilayer and a continuous partial-wetting nano-droplet at a bicrystal GB [A1]. We proposed that this series of GB complexions formed from "dewetting" of a high-order complexion (e.g., a uniform wetting film or a nanoscale equilibrium-thickness IGF) that led to a series of (uncompleted) GB transitions during cooling. This explanation is schematically illustrated in Fig. 3, and several key points are discussed below.
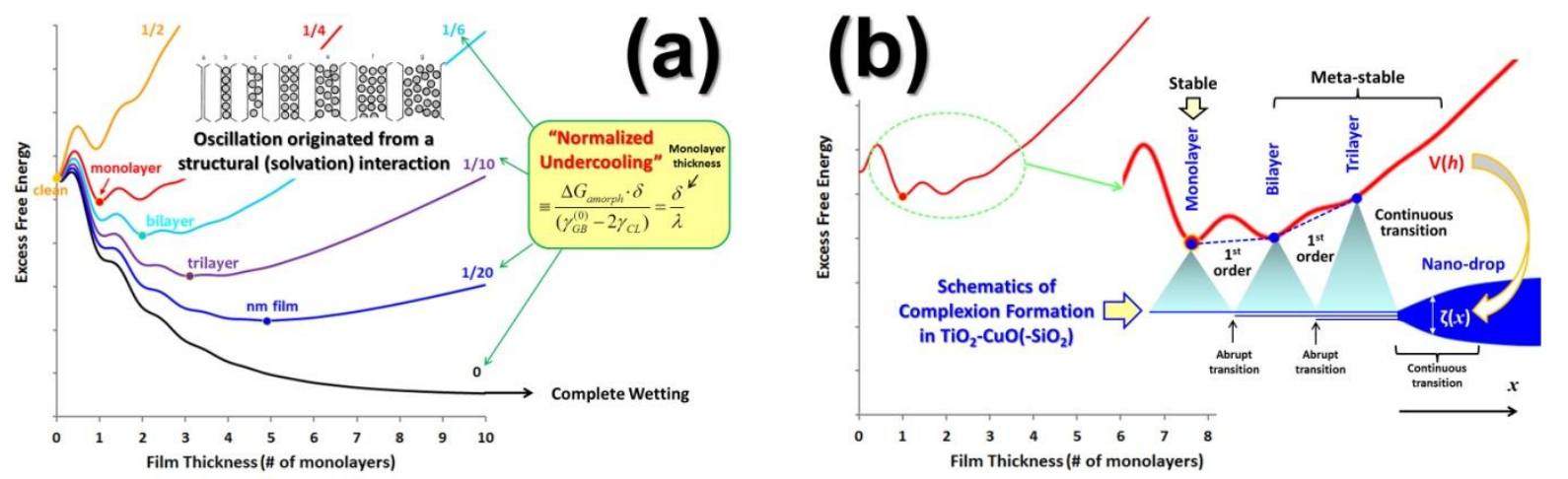

Figure 3: (a) Computed GB excess free energy vs. normalized film thickness curves. The thermodynamically-stable complexion, represented by the global minimum, is labeled for each of the normalized undercooling. With increasing normalized undercooling, a series of GB transitions may occur. (b) An explanation of the $\mathrm{GB}$ complexion formation observed in the $\mathrm{TiO}_{2}-\mathrm{CuO}-\mathrm{SiO}_{2}$ system by the Lehigh team. This series of GB complexions, which are schematically shown in the lower-right corner of panel (b), likely formed during the cooling. (Figure adapted from Ref. [A1])

The observed atomically-abrupt transition regions between the adjacent complexions suggest the interfacial transitions are generally first-order. To explain the origin of these first-order transitions (from trilayer to bilayer to monolayer sequentially), we plot the excess GB free energy $v s$. (normalized) film thickness curves in Fig. 3(a) for five different normalized undercoolings ( $\delta / \lambda$, where $\delta$ is the monolayer thickness and $\lambda$ is a thermodynamic parameter that scales the interfacial width. Here, $\lambda \equiv \Delta G_{\text {amorph }} /(-\Delta \gamma)$, where $\Delta G_{\text {amorph }}$ is the volumetric free energy to form an undercooled liquid and $-\Delta \gamma$ is the change of interfacial energies upon replacing a high-energy "dry/clean" GB with two low-energy solid-liquid interfaces; see Refs. [A1, A9] for elaboration). The minima respond to the discrete GB complexions: at each temperature (normalized undercooling), the global minimum represents the thermodynamicallystable complexion, and other minima represent meta-stable complexions.

As shown in Fig. 3(a), with reducing temperature (increasing normalized undercooling), a series of GB transitions could be produced, leading to the possible formation of wetting layer, a nanoscale IGF, a trilayer, a bilayer, a monolayer and a clean/dry GB sequentially. Fig. 3(b) schematically illustrates a 
possible explanation for the formation of a series of GB complexions during cooling. At the temperature where the interfacial structures were quenched, the monolayer was likely the stable complexion, and bilayer and trilayers were metastable. The trilayer-to-bilayer and bilayer-to-monolayer transitions are first-order, which originate from the energy barriers between the minima; these energy barriers are resulted from an oscillatory structural (solvation) interaction [A6]. Furthermore, a continuous transition from nanoscale IGF to trilayer is expected from this thermodynamic theory, and this continuous transition is represented by the continuously-changing profile at the tip of the nano-droplet, which is schematically shown in the lower-right corner of Fig. 3(b). According to the statistical model for the oscillatory structural interaction, the energy barriers that produce the first-order (layering) transitions decay exponentially, where the decaying length is equal to the monolayer thickness $(\delta)$; thus, the transition should become virtually continuous after three layers, where the barrier height decays by $1 / e^{3}$.

\section{(C) Grain Boundary Complexions and Transitions in Si-Au (Our Primary Model System)}

A major scientific goal for this project was to investigate GB transitions using doped Si as the model system. In the first two years, we conducted experiments to penetrate pre-bounded Si bicrystals with Au and $\mathrm{Al}$ based liquids; we found that the control of the native oxide on $\mathrm{Si}$ was a major challenge; oxidebased nanoscale IGFs were observed in $\underline{\mathrm{Si}}-\mathrm{Au}$ (but not $\underline{\mathrm{Si}}-\mathrm{Al})$; selected examples are shown in Fig. 4.

(a)

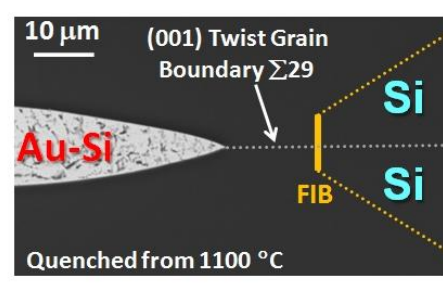

SEM

(b)

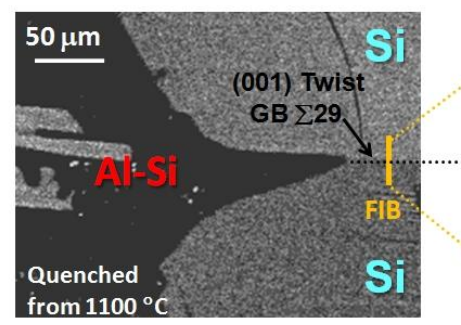

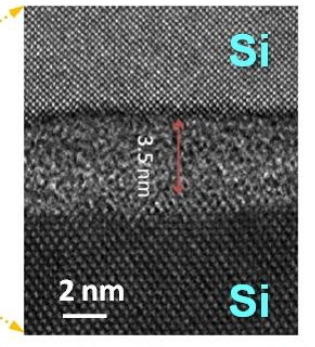

Aberration-Corrected STEM (by Lehigh collaborators)

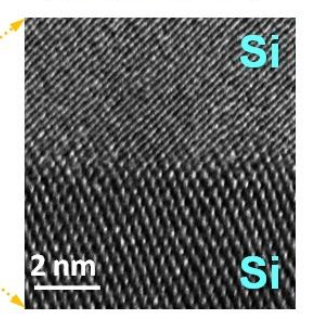

Figure 4: Representative results of the (a) $\underline{\mathrm{Si}}-\mathrm{Au}$ and (b) $\underline{\mathrm{Si}}-\mathrm{Al}$ systems from experiments conducted in the first two years, in which pre-made Si bicrystals were annealed in contact with Au or Al based liquids to allow liquids to penetrate along the GBs and form complexions. Notably, a nanoscale IGF was observed in $\underline{\mathrm{Si}}$-Au but not in Si-Al; subsequent analysis showed that this nanoscale "amorphous" IGF is oxide based. Presumably, Al served as an effective getter that reduced and eliminated oxide-based IGFs.

Subsequently, we worked with our Purdue subcontractor (Prof. Minghao Qi and co-workers) and developed a new method to make oxide-free specimens (with details reported in Ref. [A2]). Then, we

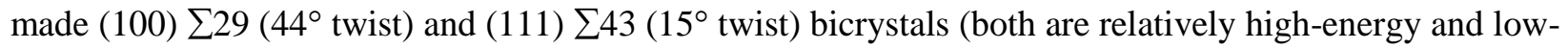
symmetry GBs; selected to represent general GBs), with thin Au layers sandwiched in between. We also made bicrystals with other metal layers, including Ti, Pt and Al. However, the most interesting results were found in the $\underline{\mathrm{Si}}-\mathrm{Au}$ system, so we focus on $\underline{\mathrm{Si}}-\mathrm{Au}$ in this report. These specimens were heated 
treated under different conditions, and we examined the GB transitions that occurred during the dewetting of the Au films upon cooling.

After specimens were prepared by us at Clemson University, the aberration-corrected high-angle annular dark field scanning transmission electron microscopy (HAADF-STEM) characterization was conducted at Lehigh University by our collaborators (Prof. Hammer and co-workers). One of the most important observations was a first-order GB transition from a bilayer to an intrinsic (nominally "clean") GB in the Si-Au system at the Si (111) $\Sigma 43$ twist GB. This observation is illustrated in Fig. 5(a). Here, the most striking finding is the co-existence of a bilayer and clean/intrinsic GB segment at the same GB with an atomically-abrupt transition, as vividly shown in middle image in Fig. 5(a). This indicates the occurrence of a bilayer to "clean" GB transition during cooling. Furthermore, the atomically-abrupt transitions between the two GB complexions [again, see the middle image Fig. 5(a)] suggests that the GB transition from a bilayer to an intrinsic/clean GB is first-order, because it is associated with a discontinuity (abrupt "jump") in the interfacial excess of the solute (Au) adsorption.

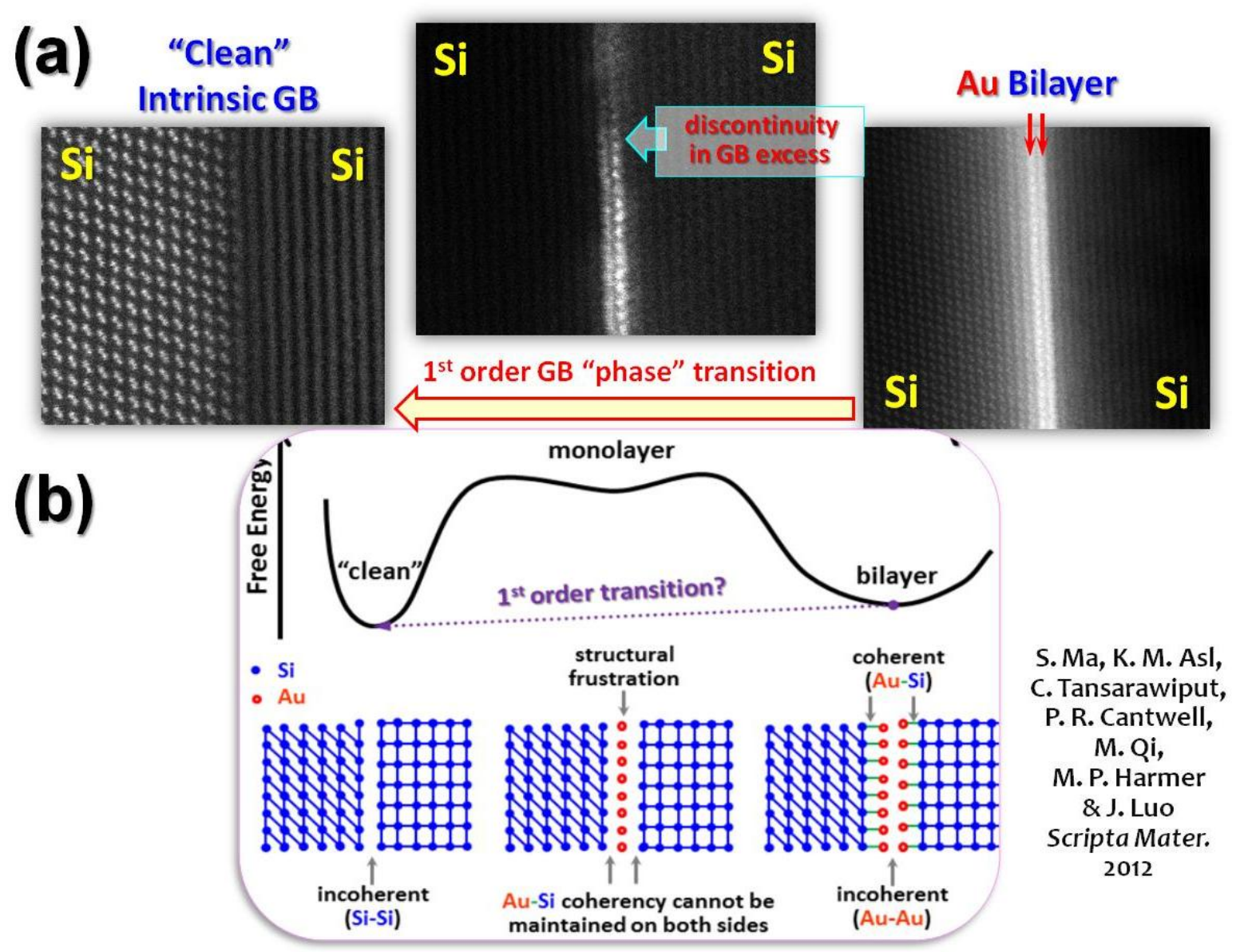

Figure 5: (a) HAADF-STEM micrograph showing the abrupt transition region between the bilayer and the "clean" GB, indicating the occurrence of a first-order GB transition between them. (b) Schematic illustration of free-energy states that may lead to this first-order transition. (Figure adapted from Ref. [A2])

We explained the bilayer stabilization and the origin of this first-order transition by using a regularsolution lattice-gas model. We used the Miedema model to show that Au-Si bonds are stronger than the average of $\mathrm{Si}-\mathrm{Si}$ and $\mathrm{Au}-\mathrm{Au}$ bonds; thus, $\mathrm{Au}$ atoms bond strongly to the $\mathrm{Si}$ atoms on the adjacent $\mathrm{Si}$ grain 
surface. As illustrated in Fig. 5(b), it is energetically expensive to form an Au monolayer at the lowsymmetry $(\Sigma 43)$ twist GB because the Au monolayer cannot grow coherently with respect to both grain surfaces. In other words, both $\mathrm{Si}$ grain surfaces would like to impose structural order onto the monolayer, which would result in a structural frustration that destabilizes the monolayer complexion. In contrast, in a bilayer, each $\mathrm{Au}$ layer can follow the order of their adjacent grain, resulting in an incoherent $\mathrm{Au}-\mathrm{Au}$ interface (which is energetically preferred over an incoherent $\mathrm{Si}$-Au interface); thus this bilayer complexion can be more stable. Furthermore, this analysis suggests that an interfacial phase transition from a bilayer to an intrinsic GB is likely first-order because of the existence of an energy barrier between the states [Fig. 5(b)], which was supported by experimental observation of an atomically-abrupt transition between the bilayer and a "clean" GB, as illustrated in the middle image in Fig. 5(a).

This observation and our model illustrate that the generic interfacial thermodynamic model discussed in $\S \mathrm{B}$ provides important insights about the physical origin of GB complexions, but it is not sufficiently realistic to describe GB complexion transitions in all systems. Specifically, a model that considers atoms as structure-less spheres cannot explain the bilayer-to-intrinsic GB transition in the absence of a monolayer [Fig. 5(a)]; on the other hand, bilayer-to-intrinsic GB transition can be well explained in a quasi-chemical model shown in Fig. 5(b) and discussed above.

Another interesting observation made for the $\mathrm{Si}$ (111) $\Sigma 43$ twist GBs is the formation of stepstabilized "ultra-nanowires", as shown in Fig. 6. HAADF STEM images have shown that some Si (111) $\Sigma 43$ twist GBs contains intermittent Au-rich "bilayer patches" separated by clean GB at the edge-on condition [Fig. 6(e)], which are actually a region of step-stabilized, bilayer-like, "ultra-nanowires" that are $\sim 2$ atoms thick and 5 to 10 atoms wide. This discovery was made during tilting experiments. When tilted to $\sim 20$ degrees, the "ultra-nanowires" [represented by the parallel lines in Fig. 6(b)] are clearly visible, as shown in Fig. 6(a)-(c). In all cases, the Au nanowires are about 2 atoms thick, 5-10 atoms wide, and extended through the entire TEM sample (suggesting that they are macroscopic in length). They are spaced periodically along the bicrystal GB. Novel applications can be envisioned.

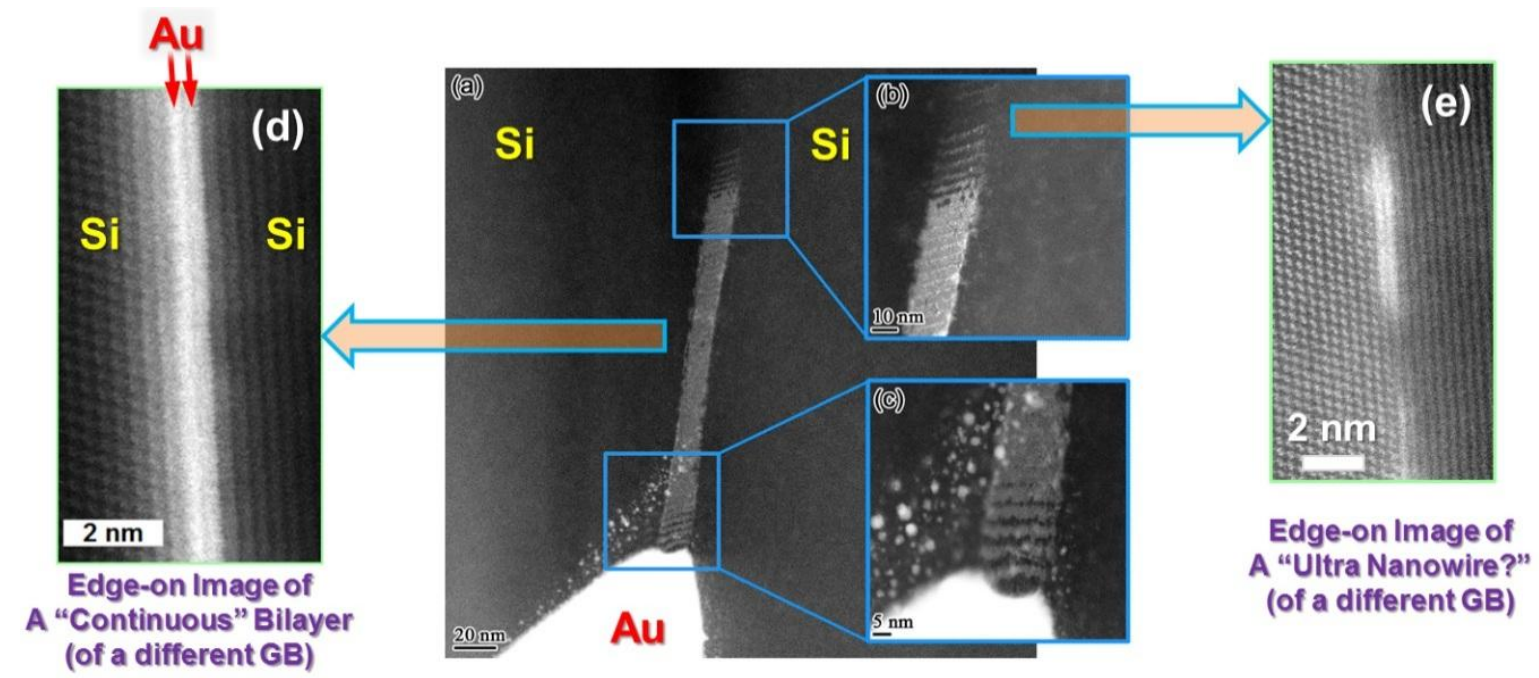

Figure 6: Panels (a)-(c) show a Si (111) twist GB ( $\left.\sum 43\right)$ tilted $20^{\circ}$ away from the edge-on orientation so that the three-dimensional structure of the Au segregation can be seen. Panel (d) shows a "continuous" bilayer at the edge-on condition, which corresponds to the middle region of (a). Some of the "bilayers patches" observed at the edge-on condition [see, e.g., Panel (e)] are in fact "ultra nanowires" that are 2 atoms thick and 5 to 10 atoms wide, which are stabilized by linear steps (ledges) at the GB. (Unpublished results; specimens made at Clemson and characterized at Lehigh.) 
Prior to the systematic investigation of $\mathrm{Si}$ (111) $\Sigma 43$ twist GBs (with the key results summarized above), we also examined the $\mathrm{Si}$ (100) $\sum 29$ twist GBs. This study was motivated by a recent atomistic simulation by Sutton and co-workers [28] that indicated that the Si (100) $\sum 29$ twist GB would undergo structural transitions starting at 0.7-0.8 $T_{\mathrm{m}}$. Since Si-Au is one of the best metallic glass forming systems, we initially hypothesized that adding Au would promote the formation of a "disordered" GB complexion. Surprisingly, the characterization showed that the Si (100) $\sum 29$ GBs in the Si-Au system only exhibited low levels of structural disorder with submonolayer adsorption of Au (Fig. 7); in these experiments, we heated the specimens up to $\sim 1 \mathrm{~K}$ below the melting temperature of $\mathrm{Si}$ (i.e., $>0.999 T_{\mathrm{m}}$ ) and gas-quenched these specimens (with the initial cooling rate being $\sim 500 \mathrm{~K}$ per minute). The most "disordered" GB structure observed for all the quenched (100) $\sum 29$ GBs that we have characterized is shown in Fig. 7.

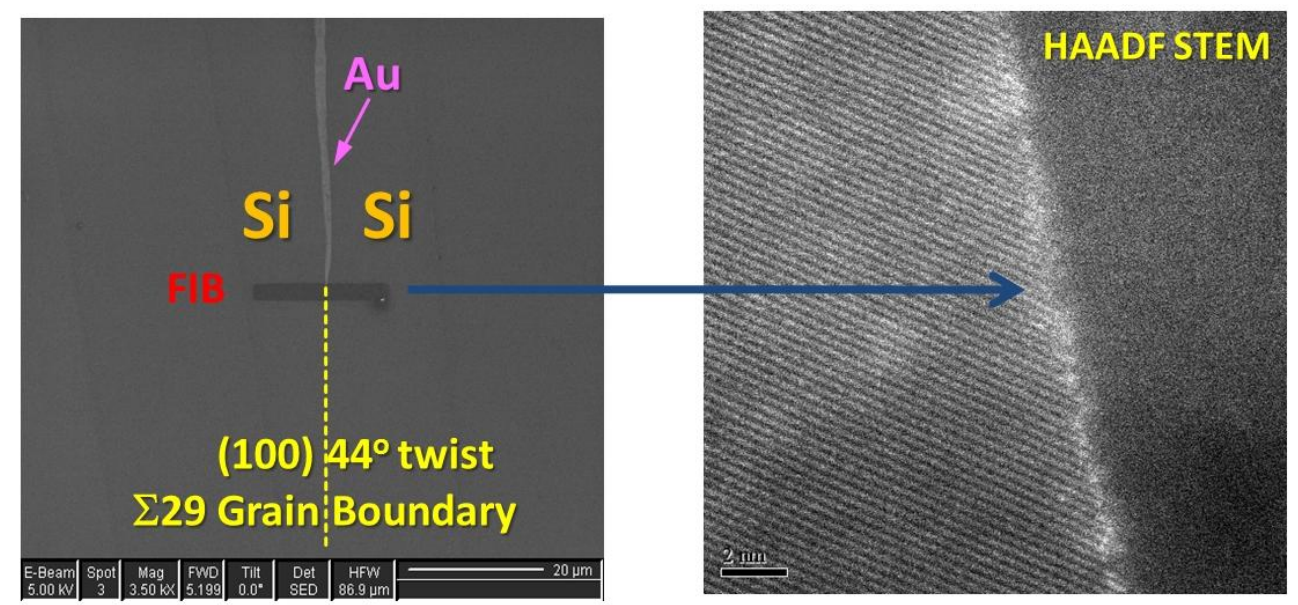

Figure 7: The most "disordered" (100) $\Sigma 29$ twist GB observed in this study, with a submonolayer level of Au adsorption visible in the HAADF-STEM micrograph. (Unpublished results; specimens made at Clemson and characterized at Lehigh.)

In all above cases, specimens were made at Clemson University with the help of our subcontractor at Purdue University (both supported by this DOE-BES grant) and characterized by aberration-corrected HAADF-STEM at Lehigh University by our collaborators, Prof. Hammer and co-workers, who were supported by a separate DOE-BES grant.

\section{(D) Grain Boundary Complexions in Ni-Bi (The "Contingent Plan" in the Original Proposal)}

In the original proposal entitled "Grain Boundary Complexions and Transitions in Doped Silicon," the PI proposed to use $\mathrm{Ni}-\mathrm{Bi}$ as a secondary model system to investigate GB complexions (in case that the results from Si systems were less ideal). Thus, we conducted an exploratory study in Ni-Bi in parallel with the $\mathrm{Si}$ study. In the Ni-Bi system, we discovered a bilayer interfacial phase of adsorbed $\mathrm{Bi}$ atoms at Ni general GBs, which is the cause of liquid metal embrittlement in this system [A4]. This example strikingly demonstrates that GBs can undergo a phase transition that cause drastic changes in properties. Furthermore, we made a single observation of a trilayer. The observations of the most controversial bilayer and trilayer interfacial phases in a simple binary metallic system (in addition to that in the $\mathrm{Si}-\mathrm{Au}$ system described above), where interpretations of both their formation mechanisms and HAADF-STEM images are less equivocal, authenticate the general existence of this series of complexions. Thus, these observations critically support the complexion theory. Furthermore, this discovery provides a new perspective to understand the atomic-level mechanisms for liquid metal embrittlement as well as the more 
general GB embrittlement phenomena in metals [A4]. This extra "sidetracked" work (that was initially planned as a "backup" experiment in the contingent plan in the original proposal) was published in Science [A4] and generated significant impacts.

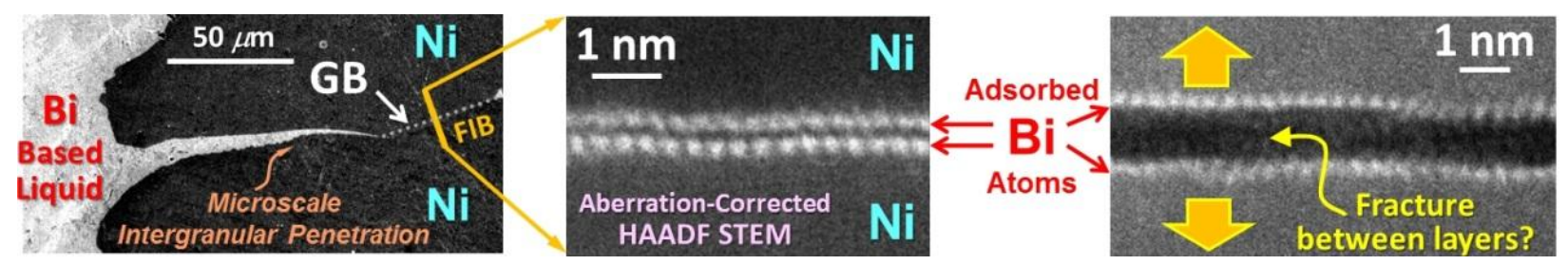

Figure 8: The observation of a bilayer interfacial phase in $\mathrm{Ni}-\mathrm{Bi}$ provided a new perspective to understand the mysterious atomic level mechanisms for liquid metal embrittlement, which have puzzled the materials and physics communities for over a century; this work was published in Science [A4].

Finally, a systematic study was conducted to investigate the kinetics of intergranular liquid metal penetration in the $\mathrm{Ni}-\mathrm{Bi}$ system in the presence of impurities (such as $\mathrm{Mn}, \mathrm{Sn}$ and $\mathrm{Fe}$ ). Our study revealed that a small amount of impurities can drastically change the GB penetration and corrosion behaviors, and we have developed a framework to systematically explain these interesting results. This study, recently published in Acta Materialia [A1], is practically important for understanding and controlling liquid metal corrosion with applications in liquid-metal cooled nuclear reactors.

\section{(E) Scientific Workforce Development}

A Clemson student, Kaveh Meshinchi Asl, has completed his thesis research with the support from this BES project, and he received his Ph.D. degree in May 2012.

\section{Publications}

[A1] S. Ma, P. R. Cantwell T. J. Pennycook, N. Zhou, M. P. Oxley, D. N. Leonard, S. J. Pennycook, J. Luo and M. P. Harmer, "Grain Boundary Complexion Transitions in $\mathrm{WO}_{3}$ - and $\mathrm{CuO}$-doped $\mathrm{TiO}_{2}$ Bicrystals," accepted for publication in Acta Materialia pending minor optional revisions

[A2] S. Ma, K. Meshinchi Asl, C. Tansarawiput, P.R. Cantwell, M. Qi, M.P. Harmer, and J. Luo, “A Grain Boundary Transition in Si-Au," Scripta Materialia, 66, 203-206 (2012)

[A3] K. M. Asl and J. Luo, "Impurity Effects on the Intergranular Liquid Bismuth Penetration in Polycrystalline Nickel,” Acta Materialia, 60, 149-165 (2012)

[A4] J. Luo, H. Cheng, K. M. Asl, C. J. Kiely, and M. P. Harmer, "The Role of a Bilayer Interfacial Phase on Liquid Metal Embrittlement," Science, 333, 1730-33 (2011)

[A5] R. H. French, V. A. Parsegian, R. Rodgornik, R. F. Rajiter, A. Jagota, J. Luo, et al., "Long Range Interactions in Nanoscale Science," (review article), Reviews of Modern Physics, 82, 1887-1944 (2010)

[A6] J. Luo, "Grain Boundary Complexions: The Interplay of Premelting, Prewetting and Multilayer Adsorption," Applied Physics Letters, 95 [7], 071911 (2009).

[A7] S. J. Dillon, M. P. Harmer, and J. Luo, "Grain Boundary Complexions in Ceramics and Metals: An Overview" (invited), JOM, 61 [12], 38-44 (December 2009). 
[A8] J. Luo, S. J. Dillon and M.P. Harmer, "Interface-Stabilized Nanoscale Quasi-Liquid Films" (overview article for non-specialists), Microscopy Today, 17 [4], 22-26, July 2009.

[A9] J. Luo, "Liquid-Like Interface Complexion: from Activated Sintering to Grain Boundary Diagrams" (invited review), Current Opinion in Solid State \& Materials Science, 12 [5/6], 81-88 (2008).

For all research articles, all Clemson authors were solely supported by this DOE-BES grant and Clemson authors acknowledged only this DOE-BES grant, contract No. DE-FG02-08ER46511 (outside collaborators who were not supported by this grant acknowledged their own supports). Some review/overview articles, which cover broader perspectives, acknowledged both this DOE-BES grant and other supports (where we typically delineated the part of work that was support by this grant, as required by DOE).

\section{Cost status}

- Approved budget: $\$ 380,000$

- Unspent funds at the end of the project: $\$ 0$

\section{Additional References (Selected key references other than publications A1-A9 listed in §6)}

[1] Hart EW. Two-Dimensional Phase Transformation in Grain Boundaries. Scripta Metallurgica 1968;2:179.

[2] Hart EW. Grain Boundary Phase Transformations. In: Hu H, editor. The Nature and Behavior of Grain Boundaries. New York: Plenum, 1972. p.155.

[3] Cahn JW. Transition and Phase Equilibria among Grain Boundary Structures. J. Phys.-Paris 1982;43:C6.

[4] Kikuchi R, Cahn JW. Grain Boundaries with Impurities in a Two-Dimensional Lattice-Gas Model. Phys. Rev. B 1987;36:418

[5] Kikuchi R, Cahn JW. Grain Boundary Melting Transition in a Two-Dimensional Lattice-Gas Model. Phys. Rev. B 1980;21:1893.

[6] Cahn JW. Critical Point Wetting. J. Chem. Phys. 1977;66:3667.

[7] Clarke DR. On the Equilibrium Thickness of Intergranular Glass Phases in Ceramic Materials. $J$. Am. Ceram. Soc. 1987;70:15.

[8] Tang M, Carter WC, Cannon RM. Grain Boundary Transitions in Binary Alloys. Phys. Rev. Lett. 2006;97:075502.

[9] Tang M, Carter WC, Cannon RM. Diffuse Interface Model for Structural Transitions of Grain Boundaries. Phys. Rev. B 2006;73:024102.

[10] Wynblatt P, Chatain D. Solid-State Wetting Transitions at Grain Boundaries. Mater.Sci. Eng. A 2008;495:119.

[11] Wynblatt P, Chatain D. Anisotropy of Segregation at Grain Boundaries and Surfaces. Metall. Mater. Trans. A 2007;38A:438.

[12] Luo J. Stabilization of Nanoscale Quasi-Liquid Interfacial Films in Inorganic Materials: A Review and Critical Assessment. Crit. Rev. Solid State Mater. Sci. 2007;32:67. 
[13] Cannon RM, Rühle M, Hoffmann MJ, French RH, Gu H, Tomsia AP, Saiz E. Adsorption and Wetting Mechanisms at Ceramic Grain Boundaries. Ceramic Transactions (Grain Boundary Engineering in Ceramics) 2000;118:427.

[14] Luo J, Gupta VK, Yoon DH, Meyer HM. Segregation-Induced Grain Boundary Premelting in Nickel-doped Tungsten. Appl. Phys. Lett. 2005;87:231902.

[15] Gupta VK, Yoon DH, Meyer III HM, Luo J. Thin Intergranular Films and Solid-State Activated Sintering in Nickel-Doped Tungsten. Acta Mater. 2007;55:3131.

[16] Shi X, Luo J. Grain boundary wetting and prewetting in Ni-doped Mo. Appl. Phys. Lett. 2009;94:251908.

[17] Avishai A, Scheu C, Kaplan WD. Intergranular Films at Metal-Ceramic Interfaces Part I - Interface Structure and Chemistry. Acta Mater. 2005;53:1559.

[18] Scheu C, Dehm G, Kaplan WD. Equilibrium Amorphous Silicon-Calcium-Oxygen Films at Interfaces in Copper-Alumina Composite Prepared by Melt Infiltration. J. Am. Ceram. Soc. 2000;84:623.

[19] Baram M, Chatain D, Kaplan WD. Nanometer-Thick Equilibrium Films: The Interface Between Thermodynmaics and Atomistics. Science 2011;332:206.

[20] Luo J, Chiang Y-M. Existence and Stability of Nanometer-Thick Disordered Films on Oxide Surfaces. Acta Mater. 2000;48:4501.

[21] Luo J, Chiang Y-M, Cannon RM. Nanometer-Thick Surficial Films in Oxides as A Case of Prewetting. Langmuir 2005;21:7358.

[22] Luo J, Chiang Y-M. Equilibrium-thickness Amorphous Films on \{11-20\} Surfaces of $\mathrm{Bi}_{2} \mathrm{O}_{3}-\mathrm{Doped}$ ZnO. J. European Ceram. Soc. 1999;19:697.

[23] Dillon SJ, Harmer MP. Multiple Grain Boundary Transitions in Ceramics: A Case Study of Alumnia. Acta Mater. 2007;55:5247.

[24] Dillon SJ, Tang M, Carter WC, Harmer MP. Complexion: A new concept for kinetic engineering in materials science. Acta Mater. 2007;55:6208.

[25] Harmer MP. Interfacial Kinetic Engineering: How Far Have We Come Since Kingery's Inaugural Sosman Address? J. Am. Ceram. Soc. 2010;93:301.

[26] Harmer MP. The Phase Behavior of Interfaces. Science 2011;332:182.

[27] Pandit R, Schick M, Wortis M. Systematics of Multilayer Adsorption Phenomena on Attractive Substrates. Phys. Rev. B 1982;26:5112.

[28] von Alfthan S, Kaski K, Sutton AP. Molecular Dynamics Simulations of Temperature-Induced Structural Transitions at Twist Boundaries in Silicon. Phys. Rev. B 2007;76:245317. 